\title{
On the potential distribution in Hall thrusters
}

\author{
Michael Keidar ${ }^{\mathrm{a})}$ and Alec D. Gallimore \\ Department of Aerospace Engineering, University of Michigan, Ann Arbor, Michigan 48109 \\ Yevgeny Raitses \\ Princeton Plasma Physics Laboratory, Princeton, New Jersey 08543 \\ lain D. Boyd \\ Department of Aerospace Engineering, University of Michigan, Ann Arbor, Michigan 48109
}

(Received 6 April 2004; accepted 4 August 2004)

\begin{abstract}
A model of the plasma flow in a Hall thruster channel is developed that takes into account the two-dimensional current conservation effect and relies on some experimental input parameters, such as magnetic field and electron temperature distribution. The model is an attempt to explain the experimentally found nonuniform potential distribution across the thruster channel. This effect is explained by the change of the electron mobility across a magnetic field due to the magnetic field gradient and due to the electron current along the magnetic field driven by the electron temperature gradient. () 2004 American Institute of Physics. [DOI: 10.1063/1.1797555]
\end{abstract}

A Hall thruster is a cross-field device in which ions are accelerated in a quasineutral plasma. Because of this feature, a Hall thruster offers much higher thrust density than other types of ion thrusters. Typically, a strong electric field is established in the Hall thruster channel region of large magnetic fields. This electric field is responsible for ion acceleration. An electron closed drift or Hall current is the direct consequence of passing electron current across a magnetic field. Consideration of only the radial component of the magnetic field leads to a one-dimensional picture of the plasma flow in the Hall thruster channel. According to conventional wisdom, the electric potential in the Hall thruster channel is governed by the magnetic field distribution in that the equipotential contours tend to line up with the magnetic field lines. This stems from the fact that the electric field tends to be zero along magnetic field lines due to high electron mobility in this direction. ${ }^{1}$ Indeed, with a correction of a logarithmic factor due to the possible density variation along the magnetic field, this is the case in most conventional Hall thruster channels (Refs. 2-5). In addition, in virtually all models, it is assumed that the secondary electrons emitted from the channel walls are thermalized with the main electron population so that the electron temperature is also constant along the magnetic field line. Note that use of this assumption will be avoided here and will be replace by measured electron temperature distribution.

However, the nonuniformity of some quantities across the dielectric channel may change the above-mentioned balance and lead to an electron temperature gradient. For instance, placing a segmented electrode with low secondary electron emission may create an electron temperature gradient between the dielectric walls. ${ }^{6}$ This electron temperature gradient may lead to electron current along the magnetic field and as a result equipotential contours may deviate from the magnetic field lines. 6 An electron temperature gradient may also be induced by other means such as by nonuniformities in the magnetic field. ${ }^{7,8}$ It was found in experiments that under certain operating conditions a distinctive "jet" po-

\footnotetext{
a) Author to whom correspondence should be addressed; electronic mail:
} keidar@engin.umich.edu

tential structure developed in the Hall thruster channel (Refs. 6-8). In other words, the potential distribution deviates from the magnetic field profile and the peak of the electric field is shifted downstream of the exit plane.

This structure may result in a significant divergence of beam ions as they are accelerated out of the thruster. It should be noted that laser-induced fluorescence (LIF) data support the evidence of the potential nonuniformity ("jet") in the radial direction. ${ }^{9}$ LIF measurements show that the ion velocity radial profile is consistent with the independently measured nonuniform potential distribution. While this abnormal potential distribution was found in several quite different Hall thrusters, it can exist only under certain conditions (Refs. 6-8). For instance, changing from high power to lower power, which results in a uniform electron temperature distribution across the channel width, ${ }^{7}$ or placing two segmented electrodes along the channel wall instead of a single electrode ${ }^{6}$ leads to the disappearance of the potential "jet" and results in a radially uniform potential distribution. ${ }^{6-8}$ In this letter we are attempting to explain the observed potential nonuniformity effect by considering the twodimensional (2D) current conservation problem in the Hall thruster channel.

Let us begin with a brief description of the plasma flow model. The plasma flow starts in the near anode region and the lateral boundary of the quasineutral plasma flow region is the entrance to the sheath at the dielectric walls [see Fig. 1(a)]. The entire quasineutral plasma region is considered as a presheath in which boundary conditions for the sheath entrance are developed. Details of the model of the plasmasheath transition region are described elsewhere. ${ }^{5}$ In this model we will assume that the magnetic field has only a radial component as shown in Fig. 1(a). However, the magnetic field strength may vary in the radial direction. A hydrodynamic model is employed in a $2 \mathrm{D}$ domain assuming that the system reaches a steady state. The momentum and mass conservation equations for ions and neutrals under these conditions have the following form:

$$
n m_{i}\left(V_{i} \nabla\right) V_{i}=n e E-\nabla P_{i}-Z_{i} n n_{a} m_{i}\left(V_{i}-V_{a}\right),
$$




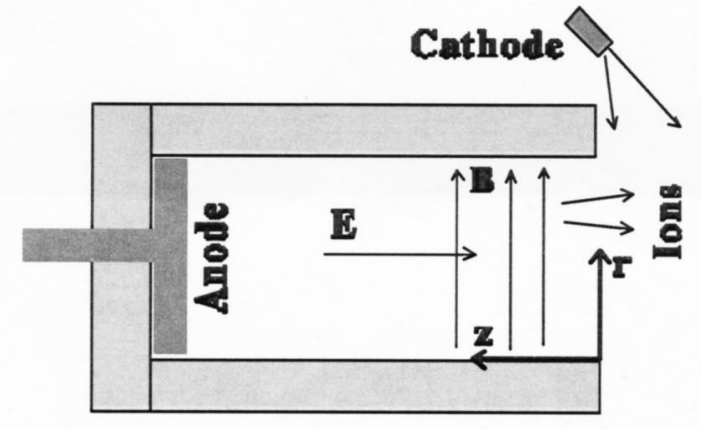

(a)

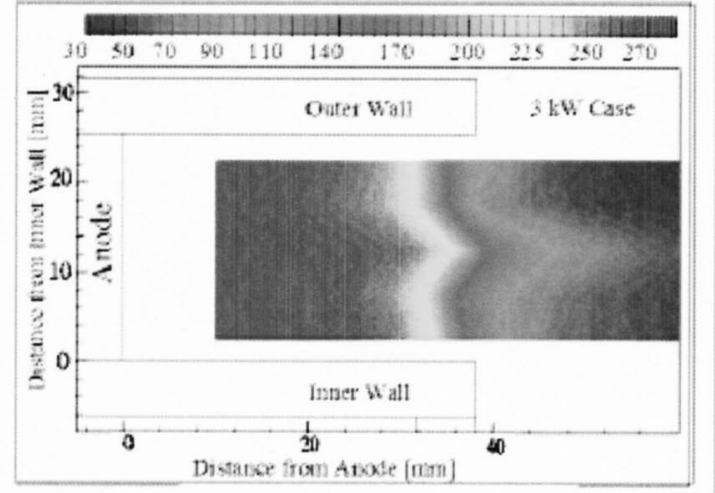

(b)

(b) Inner Segmented Electrode

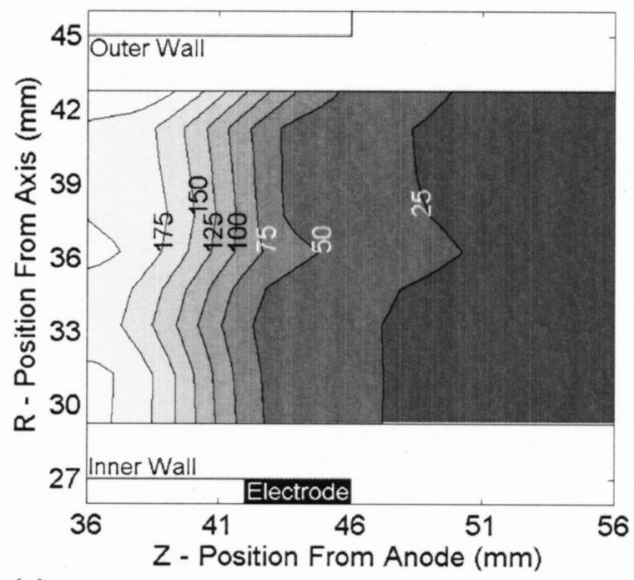

(c)

FIG. 1. Schematics of the thruster, model geometry (a) and experimental data on potential distribution in the Hall thruster channel; (b) Experimental data from Ref. 7; (c) experimental data from Ref. 6.

$$
\begin{aligned}
& \nabla\left(n V_{i}\right)=Z_{i} n n_{a}, \\
& \nabla\left(n_{a} V_{a}\right)=-Z_{i} n n_{a},
\end{aligned}
$$

where $i, a$ represent ions and neutrals, $n$ is the plasma density, $E$ is the electric field, $Z_{i}$ is the ionization rate, and $V$ is the velocity.

Since only the radial magnetic field component is considered in the model, the electron transport is much greater in the azimuthal direction $(E \times B$ drift) than in the axial direction. Usually in Hall thruster models, an assumption of a constant "thermalized" potential along the magnetic field is used (Refs. 4-6). In this model, however, we will employ a more general two-dimensional model for the electron flow instead of Boltzmann relation along the magnetic field. We consider that the electron transport across the magnetic field is due to several collision mechanisms (i.e., electron-neutral and electron-wall collisions) as well as anomalous (Bohm) diffusion with a total effective collision frequency of $\nu_{\text {ef }}$ (Refs. 4-6). Under the considered conditions, the simplified Ohm's law can be written in component form as

$$
\begin{aligned}
& j_{r}=\sigma\left(-\frac{\partial \varphi}{\partial r}+\frac{\partial T_{e}}{\partial r}+T_{e} \frac{\partial \ln n}{d r}\right), \\
& j_{z}=\frac{\sigma}{\left(1+\beta^{2}\right)}\left(-\frac{\partial \varphi}{\partial z}+\frac{\partial T_{e}}{\partial z}+T_{e} \frac{\partial \ln n}{\partial z}\right),
\end{aligned}
$$

where $\beta=e B / m_{e} \nu_{\text {ef }}$ is the Hall parameter, $T_{e}$ is the electron temperature, $\varphi$ is the potential, and $\sigma=e^{2} n / m_{e} \nu_{\text {ef }}$ is the classical plasma conductivity. In addition, current conservation implies that

$$
\frac{\partial j_{r}}{\partial r}+\frac{\partial j_{z}}{\partial z}+\frac{j_{r}}{r}=0
$$

The numerical analysis is similar to that developed previously. ${ }^{5,10}$ We use the implicit two-layer method to solve the system of equation (1)-(3). These equations are approximated by a two-layer, six-point scheme. An iterative procedure is employed similarly to Ref. 10 . The equation for potential [Eq. (6)] is solved numerically by iteration using the successive overrelaxation procedure. In order to obtain a solution of the system of equations (1)-(6) the boundary conditions must be specified. The discharge voltage (potential drop along the channel) is set to be $250 \mathrm{~V}$. Along the lateral channel walls it is assumed that $\partial \varphi / \partial r=0$. More details about the boundary conditions can be found elsewhere. ${ }^{5}$

Similar to Ref. 11, instead of employing additional assumptions for the calculation of the electron temperature distribution, we will use an experimentally measured electron temperature distribution. The electron temperature and magnetic field radial distributions are taken from the same experiment. ${ }^{7,8}$ In order to simplify the calculations we will approximate the magnetic field and the measured electron temperature distributions by the following analytical expressions:

$$
\begin{aligned}
& T_{e}=T_{e 0} \exp \left(-\frac{\alpha r}{h}\right), \\
& B=B_{0} \frac{1}{1+\gamma r},
\end{aligned}
$$

where $h$ is the channel width, $\alpha, \gamma$ are numerical coefficients obtained by fitting to experimental data, ${ }^{7} T_{e 0}$ is the maximum electron temperature, and $B_{0}$ is the maximum magnetic field strength measured in Ref. 7.

The experimental data for two different thrusters are shown in Figs. 1(b) and 1(c). These data were obtained using a similar technique, namely a high-speed probe that induced very small plasma perturbations (Refs. 6-8). One can see that in each case, the potential distribution in the channel exhibits a very similar shape having a peak of the potential 

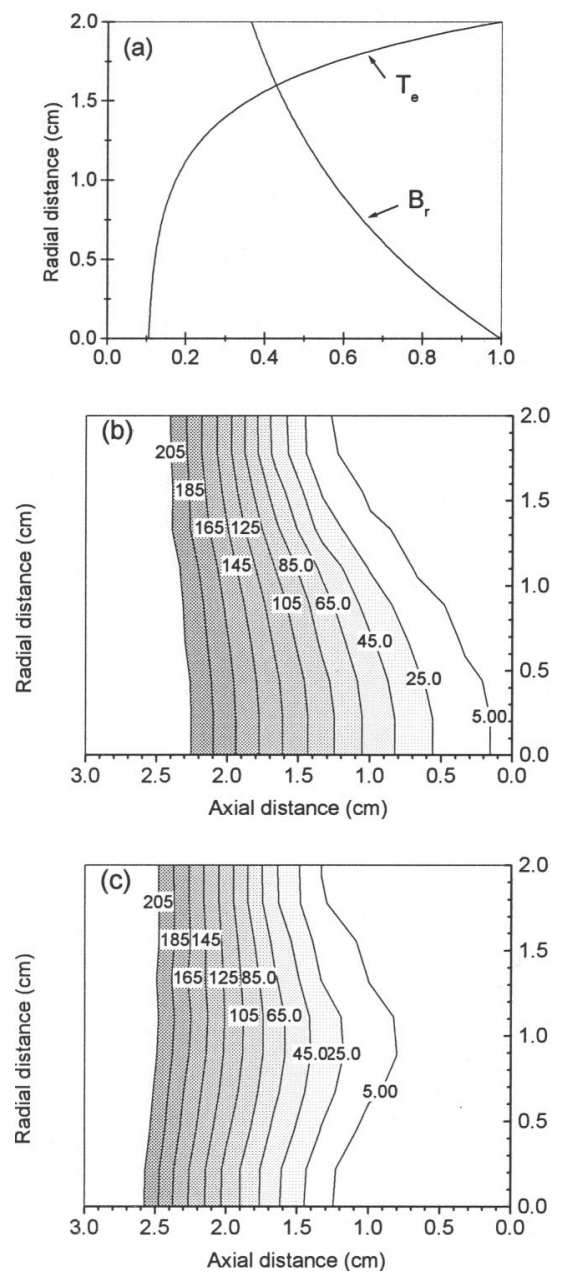

FIG. 2. (a) Electron temperature and magnetic field radial profiles according to experiment (Ref. 7). (b) Potential distribution in the case without electron temperature gradient $(\alpha=0)$. (c) Potential distribution in the case of both electron temperature and magnetic field gradients $(\alpha=7, \gamma=0.8)$.

along the channel centerline. The most interesting feature is that significantly different Hall thruster configurations produce very similar and repeatable structures.

In the following we present the calculated potential distribution in the Hall thruster channel according to Eqs. (1)-(6). These calculations are shown in Fig. 2. The radial distributions of the experimentally observed [Ref. 7, and approximated by Eqs. (7) and (8)] electron temperature and magnetic field are shown in Fig. 2(a). One can see that while the electron temperature peaks near the outer wall, the magnetic field has a maximum near the inner wall (Ref. 7).

Using the electron temperature and the magnetic field distributions as an input, the potential distribution from the current conservation equation [Eq. (6)] and plasma flow model [Eqs. (1)-(3)] was calculated. Let us first examine the effect of only a magnetic field gradient in the radial direction [Fig. 2(b)]. One can see that a nonuniform potential distribution is found with the highest electric field in the region where the magnetic field is high. This is a very much expected result that reflects the fact that the crossed field electron mobility is higher across the magnetic field in the region with a low magnetic field. On the other hand, the presence of the electron temperature gradient leads to additional nonuniformity due to the electron current along the magnetic field. Suppose the electron temperature is higher near the outer wall [see Fig. 2(a)]. In this case the current conservation requirement leads to current density increase in the middle of the channel, since high magnetic field near the inner wall prevents significant current density raise in that region. In this case the higher current density in the middle of the channel will lead to the higher electric field. Therefore the equipotential contours create a structure with a potential maximum along the middle of the channel as shown in Fig. 2(c). $1 \mathrm{t}$ can be seen that the model generally predicts the potential structure similar to the one observed experimentally. In addition, similar to the experiment this potential structure can be obtained only under certain conditions. These conditions require gradient in both magnetic field and electron temperature gradients.

In conclusion, this letter presents a model of the plasma flow in the Hall thruster channel. The model predicts the nonuniform potential distribution across the thruster channel that has been seen in experiments. This effect is explained by the change of the electron mobility across the magnetic field due to a magnetic field gradient and due to the electron current along the magnetic field driven by an electron temperature gradient. This potential structure "jet" in which the peak of the electric field is shifted downstream along the channel centerline can be found under certain conditions, namely in the presence of magnetic field and electron temperature gradients in the radial direction.

The authors thank James Haas, Tim Smith, and David Staack for very fruitful discussion. This work was partially supported by PPPL University Research Support Program.

\footnotetext{
${ }^{1}$ A. L. Morozov, Sov. Phys. Dokl. 10, 775 (1966).

${ }^{2}$ A. I. Morozov and V. V. Savelyev, in Review of Plasma Physics, edited by B. B. Kadomtsev and V. D. Shafranov (Consultant Bureau, New York, 2000), Vol. 21, p. 203.

${ }^{3}$ V. Kim, J. Propul. Power 14, 736 (1998).

${ }^{4}$ V. V. Zhurin, H. R. Kaufman, and R. S. Robinson, Plasma Sources Sci. Technol. 8, 1 (1999).

${ }^{5}$ M. Keidar, I. D. Boyd, and I. I. Beilis, Phys. Plasmas 9, 1675 (2001).

${ }^{6}$ Y. Raitses, M. Keidar, D. Staack, and N. J. Fisch, J. Appl. Phys. 92, 4906 (2002).

${ }^{7}$ J. M. Haas and A. D. Gallimore, IEEE Trans. Plasma Sci. 30, 687 (2002).

${ }^{8}$ J. M. Haas and A. D. Gallimore, Phys. Plasmas 8, 652 (2001).

${ }^{9}$ T. B. Smith, D. A. Herman, A. D. Gallimore, and R. P. Drake, IEPC-01019, 27th International Electric Propulsion Conference, Pasadena, CA, 15-19 October 2001.

${ }^{10}$ M. Keidar, I. Beilis, R. L. Boxman, and S. Goldsmith, J. Phys. D 29, 1973 (1996).

${ }^{11}$ L. Dorf, Y. Raitses, N. J. Fisch, and V. Semenov, Appl. Phys. Lett. 84, 1070 (2004).
} 